\title{
Model for the dynamics of a spherical bubble undergoing small shape oscillations between parallel soft elastic layers
}

\author{
Todd A. Hay, ${ }^{\text {a) }}$ Yurii A. llinskii, Evgenia A. Zabolotskaya, and Mark F. Hamilton \\ Applied Research Laboratories, The University of Texas at Austin, Austin, Texas 78713-8029
}

(Received 18 June 2012; revised 25 November 2012; accepted 29 November 2012)

\begin{abstract}
A model is developed for a pulsating and translating gas bubble immersed in liquid in a channel formed by two soft, thin elastic parallel layers having densities equal to that of the surrounding liquid and small, but finite, shear moduli. The bubble is nominally spherical but free to undergo small shape deformations. Shear strain in the elastic layers is estimated in a way which is valid for short, transient excitations of the system. Coupled nonlinear second-order differential equations are obtained for the shape and position of the bubble, and numerical integration of an expression for the liquid velocity at the layer interfaces yields an estimate of the elastic layer displacement. Numerical integration of the dynamical equations reveals behavior consistent with laboratory observations of acoustically excited bubbles in ex vivo vessels reported by Chen et al. [Phys. Rev. Lett. 106, 034301 (2011) and Ultrasound Med. Biol. 37, 2139-2148 (2011)].
\end{abstract} (C) 2013 Acoustical Society of America. [http://dx.doi.org/10.1121/1.4812864]

PACS number(s): 43.35.Ei, 43.40.Fz [CCC]

Pages: $1454-1462$

\section{INTRODUCTION}

The interaction of oscillating gas bubbles with nearby surfaces in liquid has been of interest for nearly a century, ${ }^{1}$ and while early work was motivated by cavitation damage on ship propellers, ${ }^{2}$ recent clinical interest in the interaction of gas or encapsulated bubbles with biological media for the purposes of imaging, gene or drug delivery, and tissue ablation has motivated many experimental and theoretical efforts. Each of these applications depends on a thorough understanding of how the dynamics of the bubbles and the tissue are related. In the present work an analytical model is developed which describes the shape and translational motion of a bubble in a channel formed by two parallel tissue-like layers. The motion of the elastic layers is also considered.

It is known theoretically, and has been confirmed experimentally, that bubbles oscillating near rigid surfaces tend to translate and form jets toward the interface, ${ }^{2,3}$ while bubbles oscillating near a free surface normally do so away from the interface (if buoyancy forces are sufficiently small). ${ }^{4}$ Between these two extremes, the translational dynamics of bubbles oscillating near compliant interfaces are much more nuanced and depend on a number of factors. ${ }^{8,9}$ With the current expanding interest in using microbubbles to induce therapeutic bioeffects in tissue there has also been interest in the deformation of, and stresses induced within, adjacent tissue due to the bubble motion. Previous experimental studies on the dynamics of cells, lipid membranes, and tissue phantoms in response to the motion of adjacent bubbles have shown that deformation and damage of the materials may occur. ${ }^{10-12}$ Several models have been proposed for compliant planar interfaces, ${ }^{13-17}$ rigid and compliant cylindrical interfaces, ${ }^{18,19}$ and other geometries. ${ }^{20-22}$ However, these

\footnotetext{
a) Author to whom correspondence should be addressed. Electronic mail: hayta@arlut.utexas.edu
}

studies have chiefly focused on the radial dynamics of the bubble, and not on the associated dynamics of the interface, or have employed numerical methods ${ }^{23-27}$ which are more flexible but less open to physical interpretation than the approach presented here.

Until recently, observations of the transient dynamics of microbubbles in tissue under conditions close to those used in a clinical setting were not available. This is due to the difficulties involved in optically observing the motion of the micron-sized bubbles inside tissue at megahertz frequencies. The first study of microbubbles in biological microvessels therefore relied on observing repeatable dynamics stroboscopically. $^{28}$ In these experiments microbubbles were observed to translate and form jets toward vessel walls, but observations of the transient dynamics of the bubbles and vessels remained elusive. Recently, however, experimental observations of the translation and jetting of microbubbles, and the accompanying vessel motion in response to transient high-amplitude acoustic excitation in an ex vivo rat mesentery model, have been reported by Chen et al. ${ }^{29,30}$ These measurements have shown that the bubbles translate and form jets toward the center of the vessel (away from the wall) and that the associated vessel displacement is typically asymmetric, with inward contraction (invagination) of the vessel typically exceeding outward distention.

The development of the present analytical model, presented in Sec. II, is motivated by the unexpected nature of these observations. While the observations of vessel motion reported in Ref. 29 are qualitatively consistent with the predictions of a modified Bernoulli relation proposed in the supplemental material, ${ }^{29}$ in the present manuscript the aim is for a physical understanding of the observed bubble dynamics, and a quantitative comparison with the observed bubble translation and vessel wall displacement. Although the geometry of the experiment (thin sections of tissue containing cylindrical vessels) differs significantly from that of the model, simulations presented in Sec. III show behavior 
which is consistent with the measurements published by Chen et al., ${ }^{29,30}$ and a quantitative agreement with measurements of vessel motion and bubble translation is obtained.

\section{THEORY}

The geometry of the problem is presented in Fig. 1. Two parallel elastic layers with shear moduli $\mu_{1}$ and $\mu_{2}$ are immersed in an incompressible liquid, positioned perpendicular to the $z$ axis, and separated by a distance $z_{2}-z_{1}$. The layers have finite thicknesses $h_{1}$ and $h_{2}$ along the $z$ axis, but extend to infinity in the $x-y$ plane. The densities of the liquid and the layers are assumed to be equal and denoted by $\rho$. A nominally spherical bubble is positioned along the $z$ axis between the two layers. Symmetry about the $z$ axis may be assumed due to the absence of any other surfaces, and the surface of the bubble relative to its local spherical coordinate system may be written as

$$
\mathbf{r}_{s}(t, \theta)=\left[s_{0}(t) P_{0}(\cos \theta)+\sum_{n=2}^{\infty} s_{n}(t) P_{n}(\cos \theta)\right] \mathbf{e}_{r}
$$

where $t$ is time, $\mathbf{e}_{r}$ is a unit vector pointing outward from the center of the bubble at an angle $\theta$ with the $z$ axis, $P_{n}$ are Legendre polynomials of order $n$, and $s_{n}(t)$ are timedependent expansion coefficients. The monopole mode amplitude $s_{0}$, hereafter denoted $R$, corresponds to radial pulsation, while the remaining amplitudes $s_{n}$ for $n \neq 1$ account for deformation of the bubble surface. The dipole mode $(n=1)$ corresponds to translation of the local coordinate system and is therefore omitted from Eq. (1) but is included in the velocity boundary condition $\partial \phi / \partial r_{s}$ presented in Eq. (19).

Lagrange's equation describing the dynamics of the system is

$$
\frac{d}{d t}\left(\frac{\partial \mathcal{L}}{\partial \dot{q}}\right)=\frac{\partial \mathcal{L}}{\partial q}
$$

where $\mathcal{L}=\mathcal{K}-\mathcal{V}$ is the Lagrangian, $\mathcal{K}$ is the kinetic energy, $\mathcal{V}$ is the potential energy, and $q$ is a generalized coordinate. In order to apply Eq. (2) we now proceed to calculate the potential and kinetic energies of the system. In calculating the energies and dynamical equations, it is assumed that the amplitudes of the surface modes are small compared to the radius, i.e., $\left|s_{n}\right| / R=O(\varepsilon) \ll 1$ for $n \geq 2$. In the present work terms up to order $\varepsilon^{2}$ are retained.

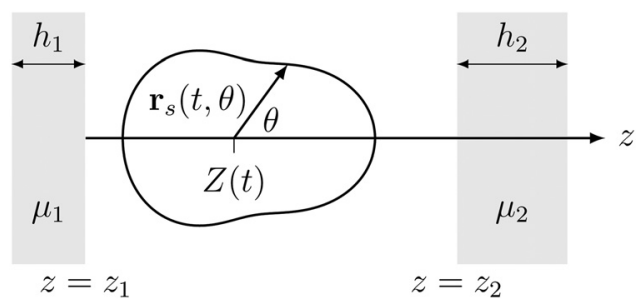

FIG. 1. Geometry and coordinate system for a spherical bubble with shape perturbations at location $z=Z(t)$ between two elastic layers with shear moduli $\mu_{1}$ and $\mu_{2}$ and thicknesses $h_{1}$ and $h_{2}$.

\section{A. Potential energy}

The potential energy consists of contributions from gas compression, surface tension, and shear strain in the elastic layers. That due to compression of the gas in the bubble is

$$
\begin{aligned}
\mathcal{V}_{g} & =V\left(P_{0}+\frac{P_{g}}{\gamma-1}\right) \\
& =\frac{4}{3} \pi R_{e}^{3}\left(P_{0}+\frac{P_{g}}{\gamma-1}\right),
\end{aligned}
$$

where $V$ is the bubble volume, $R_{e}=(3 V / 4 \pi)^{1 / 3}$ is the effective radius of the bubble, $\gamma$ is the polytropic exponent, $P_{0}$ is the ambient pressure far from the bubble, and $P_{g}$ is the pressure of the gas in the bubble. Equation (3) can be derived by recalling that $P V^{\gamma}$ is constant for an ideal gas and integrating Eq. (32) in Ref. 31. The potential energy due to surface tension is given by $\mathcal{V}_{\sigma}=\sigma S$, where $\sigma$ is surface tension, and $^{32}$

$$
\begin{aligned}
S & =4 \pi\left(R_{e}^{2}+\frac{1}{2} \sum_{n=2}^{\infty} \frac{(n-1)(n+2)}{2 n+1} s_{n}^{2}\right) \\
& =4 \pi R_{e}^{2}\left(1+\frac{2}{5} \frac{s_{2}^{2}}{R_{e}^{2}}+\frac{5}{7} \frac{s_{3}^{2}}{R_{e}^{2}}\right)
\end{aligned}
$$

is the surface area of the bubble to order $\varepsilon^{2}$.

In order to calculate potential energy due to shear strain in the elastic layers consider the displacement of a liquid element initially located at distance $\tilde{r}$ from the bubble center when the bubble has volume $\tilde{V}$. If the bubble volume changes to $V$ this liquid element will be displaced to position $r$, defined by the relationship

$$
\frac{4 \pi}{3} r^{3}-\frac{4 \pi}{3} \tilde{r}^{3}=V-\tilde{V} .
$$

In other words, the coordinates of the liquid element $x_{i}$ (at bubble volume $V$ ) may be related to the coordinates $\tilde{x}_{i}$ (at volume $\tilde{V}$ ) by ${ }^{33}$

$$
x_{i}=\frac{r}{\tilde{r}} \tilde{x}_{i}=\frac{1}{\tilde{r}}\left(\tilde{r}^{3}+\Delta\right)^{1 / 3} \tilde{x}_{i},
$$

where

$$
\Delta=\frac{3}{4 \pi}(V-\tilde{V})
$$

with $r$ expressed in terms of $\tilde{r}$ and $\Delta$ via Eq. (5).

After application of Eq. (6) the Cauchy-Green deformation tensor,

$$
G_{j j^{\prime}}=\frac{\partial x_{k}}{\partial \tilde{x}_{j}} \frac{\partial x_{l}}{\partial \tilde{x}_{j^{\prime}}} \delta_{k l},
$$

may be expressed as

$$
G_{j j^{\prime}}=f_{0}^{2}(\tilde{r}) \delta_{j j^{\prime}}+\frac{\tilde{x}_{k} \tilde{x}_{j^{\prime}}}{\tilde{r}^{2}}\left[f_{1}^{2}(\tilde{r})+2 f_{0}(\tilde{r}) f_{1}(\tilde{r})\right],
$$

where $\delta_{j j^{\prime}}$ is the Kronecker delta function, 


$$
\begin{aligned}
& f_{0}(\tilde{r})=\frac{1}{\tilde{r}}\left(\tilde{r}^{3}+\Delta\right)^{1 / 3} \\
& f_{1}(\tilde{r})=\tilde{r} \frac{d f_{0}(\tilde{r})}{d \tilde{r}}=-\frac{\Delta}{\tilde{r}\left(\tilde{r}^{3}+\Delta\right)^{2 / 3}} .
\end{aligned}
$$

With Eq. (9) in hand, the Lagrangian strain tensor $E_{j j^{\prime}}=\frac{1}{2}\left(G_{j j^{\prime}}-\delta_{j j^{\prime}}\right)$ is expressed as

$$
E_{j j^{\prime}}=\frac{1}{2}\left\{\left[f_{0}^{2}(\tilde{r})-1\right] \delta_{j j^{\prime}}+\frac{\tilde{x}_{j} \tilde{x}_{j^{\prime}}}{\tilde{r}^{2}}\left[f_{1}^{2}(\tilde{r})+2 f_{0}(\tilde{r}) f_{1}(\tilde{r})\right]\right\},
$$

and the internal strain energy density in the $j$ th elastic layer may be calculated. In spherical coordinates the relevant components of the strain tensor are

$$
\begin{aligned}
E_{r r} & =\frac{1}{2}\left[f_{0}^{2}(\tilde{r})-1+f_{1}^{2}(\tilde{r})+2 f_{0}(\tilde{r}) f_{1}(\tilde{r})\right] \\
& \simeq-\frac{2}{3} \frac{\Delta}{\tilde{r}_{3}}+O\left(\Delta^{2}\right), \\
E_{\theta \theta} & =\frac{1}{2}\left[f_{0}^{2}(\tilde{r})-1\right] \simeq \frac{1}{3} \frac{\Delta}{\tilde{r}^{3}}+O\left(\Delta^{2}\right), \\
E_{\phi \phi} & =\frac{1}{2}\left[f_{0}^{2}(\tilde{r})-1\right] \simeq \frac{1}{3} \frac{\Delta}{\tilde{r}^{3}}+O\left(\Delta^{2}\right),
\end{aligned}
$$

and therefore the energy density is

$$
\begin{aligned}
w_{j} & =\mu_{j}\left(E_{r r}^{2}+E_{\theta \theta}^{2}+E_{\phi \phi}^{2}\right) \\
& =\frac{2 \mu_{j}}{3}\left\{\frac{R_{e}^{3}}{\left[\kappa^{2}+\left(Z-z_{j}\right)^{2}\right]^{3 / 2}}-\frac{R_{0}^{3}}{\left[\kappa^{2}+\left(Z_{0}-z_{j}\right)^{2}\right]^{3 / 2}}\right\}^{2},
\end{aligned}
$$

for $j=1,2$, where $\kappa^{2}=\tilde{x}^{2}+\tilde{y}^{2}, Z_{0}$ is the initial position of the bubble, and the definitions $V=\frac{4}{3} \pi R_{e}^{3}$ and $\tilde{V}=\frac{4}{3} \pi R_{0}^{3}$ have been used. Equations (13)-(15) are valid when the distance between the bubble center and the layers is much greater than the bubble radius. For very small offset distances the order $\Delta^{2}$ terms should be included, but these terms are negligible for the cases considered here. The potential energy in the $j$ th layer is obtained by integrating the strain energy density $w_{j}$ over the volume $V_{j}$ of the layer, i.e.,

$$
\begin{aligned}
\mathcal{V}_{j}= & \int_{V_{j}} w_{j} d V_{j}=2 \pi h_{j} \int_{0}^{\infty} w_{j} \kappa d \kappa \\
= & \frac{\pi \mu_{j} h_{j}}{3}\left[\frac{R_{e}^{6}}{\left(Z-z_{j}\right)^{4}}\right. \\
& \left.-\frac{8 R_{e}^{3} R_{0}^{3}}{\left(Z_{0}-z_{j}\right)\left(Z-z_{j}\right)\left(Z+Z_{0}+2 z_{j}\right)^{2}}+\frac{R_{0}^{6}}{\left(Z_{0}-z_{j}\right)^{4}}\right] .
\end{aligned}
$$

Note that if losses are ignored, the force acting on the $j$ th layer interface may be estimated as $\mathbf{F}_{j}=-\boldsymbol{\nabla} \mathcal{V}_{j}$.

Equation (17) is valid if the layers are sufficiently thin such that the integrand $w_{j}$ can be considered constant over the thickness of the layer. A Taylor series expansion of the integrand shows that this condition will be satisfied if $3 h_{j} /\left|Z-z_{j}\right| \ll 1$. It is also worthwhile to note that contributions from shear and bending wave motion are neglected in this model. Doing so is justified for short, transient excitations because the bubble dynamics occur on a much shorter time scale than the elastodynamics of the tissue. To be more precise, the characteristic time scale of the bubble motion is approximately $t_{b} \simeq R_{0} \sqrt{\rho / P_{g}}$, where $P_{g}$ is the gas pressure in the bubble. The characteristic time scale for shear waves is $t_{s} \simeq\left|Z-z_{j}\right| / c_{t}=\left|Z-z_{j}\right| \sqrt{\rho / \mu_{j}}$, where $c_{t}=\sqrt{\mu_{j} / \rho}$ is the shear wave speed. A quasistatic condition $t_{b} / t_{s}=R_{0} \sqrt{\mu_{j} / P_{g}} /\left|Z-z_{j}\right| \ll 1$, for which the elastodynamics of the layers may be neglected, is satisfied for typical soft tissue parameters and bubble offsets (e.g., $\mu_{j} \leq 10 \mathrm{kPa}$ and $R_{0} /\left|Z-z_{j}\right| \leq \frac{1}{3}$ ), in which case $t_{b} / t_{s} \leq 0.1$.

With the shear strain potential energy calculated the total potential energy of the system may now be written as

$$
\mathcal{V}=\mathcal{V}_{g}+\mathcal{V}_{\sigma}+\mathcal{V}_{1}+\mathcal{V}_{2}
$$

\section{B. Kinetic energy}

The motion of both the fluid and the elastic layers contributes to the kinetic energy of the system. However, since the layers are assumed to be incompressible with density equal to that of the surrounding fluid, the kinetic energy of both the fluid and the layers may be calculated by considering the motion of an unbounded irrotational and incompressible fluid surrounding the bubble. The velocity potential $\phi$ of an incompressible and irrotational liquid must satisfy Laplace's equation, and the application of Green's theorem allows the kinetic energy to be written ${ }^{32}$

$$
\begin{aligned}
\mathcal{K} & =-\frac{\rho}{2} \int_{s} \phi \frac{\partial \phi}{\partial r_{s}} d S \\
& =-\pi \rho \int_{0}^{\pi} \phi\left[\dot{r}_{s}+U P_{1}(\cos \theta)\right] r_{s}^{2} \sin \theta d \theta,
\end{aligned}
$$

where the overdot denotes differentiation with respect to time, and $U=\dot{Z}$ is the translational velocity of the bubble. The velocity potential of the fluid may be expressed as

$$
\phi=\sum_{n=0}^{\infty} \frac{a_{n} R^{n+1}}{r^{n+1}} P_{n}(\cos \theta)
$$

where the coefficients $a_{n}$ are given in Ref. 34, and repeated here for convenience:

$$
\begin{aligned}
a_{0}= & H_{0}, \\
a_{1}= & -\frac{1}{2} R^{3} U+\frac{3}{10} R^{2} s_{2} U+H_{1}, \\
a_{n}= & -\frac{R^{n+1}}{n+1}\left(2 s_{n} \dot{R}+\dot{s}_{n} R\right) \\
& +\frac{3 n R^{n+1} U}{2}\left[\frac{s_{n+1}}{2 n+3}-\left(1-\delta_{2 n}\right) \frac{s_{n-1}}{2 n-1}\right]+H_{n} .
\end{aligned}
$$

In Eqs. (21)-(23) 


$$
\begin{aligned}
H_{n}= & \frac{R^{n}}{n+1} \sum_{l, m=2}^{\infty}\left\{\dot{R}\left[\frac{2 D_{n}(l, m)}{l+1}-(2 l+1) C_{n}(l, m)\right] s_{l} s_{m}\right. \\
& \left.+R\left[\frac{D_{n}(l, m)}{l+1}-(l+2) C_{n}(l, m)\right] \dot{s}_{l} s_{m}\right\}
\end{aligned}
$$

$C_{l}(m, n)$ is related to the Clebsch-Gordan coefficient $(n m 00 \mid n m l 0)$ as $C_{l}(m, n)=(n m 00 \mid n m l 0)^{2}$, and

$$
\begin{aligned}
D_{l}(m, n)= & \frac{n(n+1)}{2 n+1} \sum_{k=1}^{M}(2 m-4 k+2) \\
& \times\left[C_{l}(n-1, m-2 k+1)\right. \\
& \left.-C_{l}(n+1, m-2 k+1)\right],
\end{aligned}
$$

where $M=m / 2$ for $m$ even and $(m+1) / 2$ for $m$ odd.

The kinetic energy may now be calculated by substituting Eq. (20) into Eq. (19) and integrating over $\theta$. The focus of the present work is a qualitative description of bubble shape deformation, including the onset of jetting. Accounting for the latter requires retention of at least one spherical harmonic in Eq. (1) that is antisymmetric about $\theta=\pi / 2$, the first of which is the octupole mode $(n=3)$. Therefore Eqs. (1) and (20) are truncated at $n=3$ to obtain, after substitution in Eq. (19),

$$
\begin{aligned}
\mathcal{K}= & 2 \pi R^{2}\left[R \dot{R}^{2}+\frac{1}{6} R U^{2}+R\left(\frac{\dot{s}_{2}^{2}}{15}+\frac{\dot{s}_{3}^{2}}{28}\right)-\frac{U^{2}}{10} s_{2}\right. \\
& +\dot{R}\left(\frac{2}{3} s_{2} \dot{s}_{2}+\frac{3}{7} s_{3} \dot{s}_{3}\right)-\frac{U}{35}\left(2 s_{3} \dot{s}_{2}+\frac{3}{2} s_{2} \dot{s}_{3}\right) \\
& \left.+\frac{s_{2}^{2}}{5 R}\left(\frac{1}{3} \dot{R}^{2}+\frac{27}{35} U^{2}\right)+\frac{s_{3} U}{35 R}\left(11 s_{2} \dot{R}+\frac{9}{7} s_{3} U\right)\right],
\end{aligned}
$$

for the kinetic energy to order $\epsilon^{2}$. Since the potential energy is expressed in terms of the effective radius the kinetic energy should also be expressed in terms of $R_{e}$. To this end, the expression for the bubble volume,

$$
\begin{aligned}
V & =\frac{2 \pi}{3} \int_{0}^{\pi} r_{s}^{3} \sin \theta d \theta \\
& =\frac{4 \pi}{3} R^{3}\left[1+\frac{3}{R^{2}}\left(\frac{s_{2}^{2}}{5}+\frac{s_{3}^{2}}{7}\right)\right]+O\left(\epsilon^{3}\right),
\end{aligned}
$$

may be used to express the monopole amplitude $R$ to order $\epsilon^{2}$ in terms of the effective radius as ${ }^{32}$

$$
\begin{aligned}
R & =R_{e}-\frac{1}{R_{e}} \sum_{n=2}^{\infty} \frac{s_{n}^{2}}{2 n+1} \\
& =R_{e}-\frac{1}{R_{e}}\left(\frac{s_{2}^{2}}{5}+\frac{s_{3}^{2}}{7}\right),
\end{aligned}
$$

which is substituted into Eq. (26).

\section{Dynamical equations}

With the kinetic and potential energies in hand the Lagrangian $\mathcal{L}=\mathcal{K}-\mathcal{V}$ may be calculated, and Eq. (2) applied to obtain the dynamical equations. The dynamical equation describing the effective radius of the bubble is obtained by substituting $q=R_{e}$ into Eq. (2), yielding

$$
\begin{aligned}
R_{e} \ddot{R}_{e}+\frac{3}{2} \dot{R}_{e}^{2}= & \frac{P_{l}}{\rho}+\frac{1}{4} U^{2}+\frac{1}{2}\left(\frac{s_{2}^{2}}{3}+\frac{s_{3}^{2}}{4}\right)+\frac{s_{2} \ddot{s}_{2}}{15}+\frac{s_{3} \ddot{s}_{3}}{14}+\frac{\ddot{R}_{e}}{R_{e}}\left(\frac{2}{15} s_{2}^{2}+\frac{1}{7} s_{3}^{2}\right) \\
& +\frac{\dot{R}_{e}^{2}}{R_{e}^{2}}\left(\frac{s_{2}^{2}}{15}+\frac{s_{3}^{2}}{14}\right)+\frac{2 \dot{R}_{e}}{R_{e}}\left(\frac{2}{15} s_{2} \dot{s}_{2}+\frac{s_{3} \dot{s}_{3}}{7}\right)-\frac{11}{70} \frac{s_{2} s_{3}}{R_{e}} \dot{U} \\
& -\frac{U}{R_{e}}\left(\frac{1}{5} s_{2} \dot{s}_{3}+\frac{3}{14} \dot{s}_{2} s_{3}\right)-\frac{U^{2}}{10 R_{e}}\left(s_{2}+\frac{17}{98} \frac{s_{3}^{2}}{R_{e}}-\frac{19}{70} \frac{s_{2}^{2}}{R_{e}}\right) \\
& -\sum_{j=1}^{2} \frac{\beta_{j}}{2}\left[\frac{R_{e}^{3}}{\left(Z-z_{j}^{3}\right)^{4}}-\frac{\left.Z_{0}^{3}-z_{j}\right)\left(Z-z_{j}\right)\left(Z_{0}+Z-2 z_{j}\right)^{2}}{\left(Z_{0}\right.}\right],
\end{aligned}
$$

where $\beta_{j}=\mu_{j} h_{j} / \rho$ is a parameter depending only on the material properties and thickness of each elastic layer. The pressure $P_{l}$ in the liquid at the bubble wall is

$$
P_{l}=\left(P_{0}+\frac{2 \sigma}{R_{0}}\right)\left(\frac{R_{0}}{R_{e}}\right)^{3 \gamma}-\frac{2 \sigma}{R_{e}}-4 \nu \frac{\dot{R}_{e}}{R_{e}}-p_{\mathrm{ac}}(t),
$$

where $\nu$ is the kinematic shear viscosity of the liquid, and $p_{\text {ac }}$ is an external acoustic pressure. Models for the dynamics of encapsulated ultrasound contrast agents are readily obtained by substituting an appropriate expression for $P_{l}$ accounting for the bubble shell ${ }^{35-37}$ into Eq. (30). Viscous dissipation is taken into account for the effective radius by the term proportional to $\nu$ in Eq. (31), and is included ad hoc.

The dynamical equation for translation is obtained by substituting $q=Z$ in Eq. (2), which gives

$$
2 \pi \rho R_{e}^{2}\left(\frac{1}{3} R_{e} \dot{U}+\dot{R}_{e} U\right)=F,
$$


where

$$
\begin{aligned}
F= & 2 \pi \rho\left\{-6 \nu R_{e} U+\frac{R_{e}^{2}}{5}\left(s_{2} \dot{U}+\dot{s}_{2} U+\frac{3}{14} s_{2} \ddot{s}_{3}+\frac{2}{7} s_{3} \ddot{s}_{2}+\frac{1}{2} \dot{s}_{2} \dot{s}_{3}\right)-\frac{\dot{U} R_{e}}{35}\left(\frac{19}{5} s_{2}^{2}+\frac{17}{7} s_{3}^{2}\right)\right. \\
& +\frac{2 U R_{e}}{5}\left(s_{2} \dot{R}_{e}+\frac{17}{49} s_{3} \dot{s}_{3}-\frac{19}{35} s_{2} \dot{s}_{2}\right)-\frac{\dot{R}_{e} U}{35}\left(\frac{19}{5} s_{2}^{2}-\frac{17}{7} s_{3}^{2}\right)-\frac{11}{35} s_{2} s_{3} R_{e}\left(\ddot{R}_{e}+\frac{\dot{R}_{e}^{2}}{R_{e}}\right)-\frac{\dot{R}_{e} R_{e}}{5}\left(\frac{8}{7} s_{2} \dot{s}_{3}+\dot{s}_{2} s_{3}\right) \\
& \left.+\frac{2 R_{e}^{3}}{3} \sum_{j=1}^{2} \frac{\beta_{j}}{\left(Z-z_{j}\right)^{2}}\left[\frac{R_{e}^{3}}{\left(Z-z_{j}\right)^{3}}-\frac{2 R_{0}^{3}\left(Z_{0}+3 Z-4 z_{j}\right)}{\left(Z_{0}-z_{j}\right)\left(Z_{0}+Z-2 z_{j}\right)^{3}}\right]\right\}
\end{aligned}
$$

is the translational force. The first term in Eq. (33), which is proportional to $\nu$, accounts for the drag force on a sphere with a time-dependent radius, and is added after application of Eq. (2). Formally, this expression for the drag force is only valid for $\operatorname{Re} \ll 1$ or $\operatorname{Re} \mathcal{U} \ll 1,{ }^{38,39}$ where $\operatorname{Re}$ $=R_{e}|U| / \nu$ and $\mathcal{U}=\left|\dot{R}_{e} / U\right|$ are the Reynolds number and ratio of radial to translational velocities of the bubble, respectively. However, this expression has been shown to be accurate for bubbles oscillating at megahertz frequencies ${ }^{38}$ and it is therefore used for all simulations considered here. The primary Bjerknes force is neglected due to the fact that in the experiments the axis of the transducer was positioned perpendicular to the image plane, and therefore does not contribute to the observed motion.

The dynamical equation for the quadrupole mode is obtained by substituting $q=s_{2}$ in Eq. (2). The result, once arranged in the canonical form of a harmonic oscillator, is

$$
\begin{aligned}
\ddot{s}_{2} & +\frac{1}{R_{e}}\left[3 \dot{R}_{e}+\frac{8 \nu}{R_{e}}\left(5-16 \frac{\delta_{2}}{R_{e}}\right)\right] \dot{s}_{2} \\
& -\frac{1}{R_{e}}\left[\ddot{R}_{e}+\frac{57}{10} \frac{U^{2}}{R_{e}}-\frac{12 \sigma}{\rho R_{e}^{2}}-8 \frac{\nu \dot{R}_{e}}{R_{e}^{2}}\left(1+4 \frac{\delta_{2}}{R_{e}}\right)\right] s_{2} \\
& =\frac{3}{7 R_{e}}\left[\left(1+\frac{15}{2} \frac{s_{3}}{R_{e}} \dot{R}_{e}\right) s_{3} \dot{U}+\frac{1}{4} \dot{s}_{3} U\right]-\frac{3}{4} \frac{U^{2}}{R},
\end{aligned}
$$

where $\delta_{2}$ is a boundary layer thickness associated with vorticity, ${ }^{40}$ and the term proportional to $\nu$ accounts for damping and is added after application of Eq. (2), following Ref. 40. Assuming that vorticity generated by viscous stresses remains local to the surface of the bubble in a small boundary layer, viscous losses may be included in a straightforward manner to order $\delta \epsilon$, where $\delta$ is the ratio of the boundary layer thickness to the bubble radius. ${ }^{40}$

Setting $q=s_{3}$ in Eq. (2) gives the dynamical equation for the octupole mode:

$$
\begin{aligned}
\ddot{s}_{3} & +\frac{1}{R_{e}}\left[3 \dot{R}_{e}+\frac{10 \nu}{R_{e}}\left(7-\frac{30 \delta_{3}}{R_{e}}\right)\right] \dot{s}_{3} \\
& +\frac{2}{R_{e}}\left[\frac{17 U^{2}}{35} \frac{\ddot{R}_{e}}{R_{e}}+\frac{20 \sigma}{\rho R_{e}^{2}}+\frac{10 \nu \dot{R}_{e}}{R^{2}}\left(1+6 \frac{\delta_{3}}{R_{e}}\right)\right] s_{3} \\
& =\frac{3 s_{2}}{5 R_{e}} \dot{U}+\frac{28 s_{2}}{5 R_{e}^{2}} \dot{R}_{e} U-\frac{\dot{s}_{2} U}{5 R_{e}},
\end{aligned}
$$

where $\delta_{3}$ is another boundary layer thickness associated with vorticity. The boundary layer thicknesses $\delta_{2}$ and $\delta_{3}$ in Eqs. (34) and (35) are approximated as ${ }^{40}$

$$
\delta_{n}=\min \left(\sqrt{\frac{\nu}{\omega}}, \frac{R_{0}}{2 n}\right) .
$$

Note that the dynamical equations for the surface modes, Eqs. (34) and (35), do not contain terms involving $\beta_{j}$, and therefore do not depend directly on the presence of the elastic layers. Deformation of the bubble surface is driven by the translational motion of the bubble, which in turn depends on shear strain energy stored in the elastic layers.

\section{Layer displacement}

The velocity potential due to radial and translational motion in the vicinity of the bubble is

$$
\phi=-\frac{R_{e}^{2} \dot{R}_{e}}{r}-\frac{R_{e}^{3}}{2 r^{3}}(\mathbf{U} \cdot \mathbf{r})
$$

where $\mathbf{r}$ is a vector pointing outward from the bubble center. Therefore, the $i$ th component of the velocity vector for a point located on the surface of the $j$ th layer is given by

$$
\begin{aligned}
v_{i}= & (\boldsymbol{\nabla} \phi)_{i}=\frac{R_{e}^{2} \dot{R}_{e}}{r_{j}^{3}}\left[x_{i}-\left(Z-z_{j}\right) \delta_{i z}\right] \\
& -\frac{R_{e}^{3} U}{2 r_{j}^{3}}\left\{\delta_{i z}-\frac{3}{r_{j}^{2}}\left[x_{i}-\left(Z-z_{j}\right) \delta_{i z}\right]\left[z-\left(Z-z_{j}\right)\right]\right\},
\end{aligned}
$$

where $r_{j}$ is the distance from the bubble center to the point on the $j$ th layer surface. Equation (38) may be integrated numerically to obtain an estimate of the layer displacement.

\section{SIMULATIONS}

Simulation parameters are selected to correspond to a gas bubble oscillating in a blood vessel. Following Ref. 21 the viscosity and surface tension of the liquid are set to $\eta=5 \mathrm{mPa} \cdot \mathrm{s}$ and $\sigma=0.06 \mathrm{~N} / \mathrm{m}$. Figures 2 and 3 show the dynamics of a bubble with equilibrium radius $1.5 \mu \mathrm{m}$, initially placed a distance $Z_{0}-z_{1}=1.5 R_{0}$ from the left layer interface in a channel of width $z_{2}-z_{1}=5 R_{0}$, and driven by 

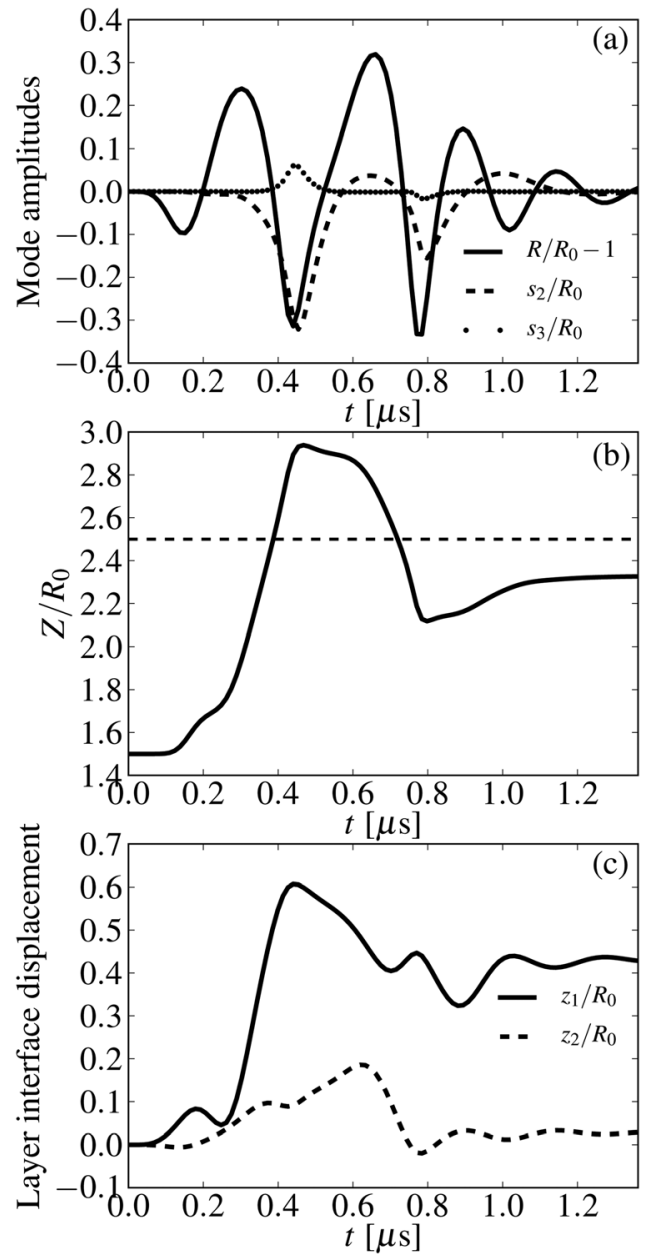

FIG. 2. Mode amplitudes (a), position (b), and displacement of the adjacent elastic layers (c) for a bubble with equilibrium radius $R_{0}=1.5 \mu \mathrm{m}$ driven by five cycles of an external sinusoidal pressure source with amplitude $330 \mathrm{kPa}$ and frequency $2.9 \mathrm{MHz}$. The bubble is initially at rest and positioned at $z=1.5 R_{0}$ in a channel of width $5 R_{0}$ formed by two elastic layers with $\beta_{1}=\beta_{2}=1 \mu \mathrm{m}^{3} / \mu \mathrm{s}^{2}$. Displacements $z_{1}$ and $z_{2}$ in part (c) correspond to the black and white dots, respectively, shown in Fig. 3.

2.5 cycles of an external sinusoidal acoustic pressure source $\left[p_{\mathrm{ac}}(t)\right.$ in Eq. (31)] with amplitude $330 \mathrm{kPa}$ and frequency $2.9 \mathrm{MHz}$ (the natural frequency of the bubble). The value of $\beta$ for the vessel walls (elastic layers) is $\beta_{1}=\beta_{2}=1 \mu \mathrm{m}^{3} /$ $\mu \mathrm{s}^{2}$. Figure 2(a) shows the time evolution of the amplitudes of the effective radius, quadrupole and octupole modes. The position of the bubble is shown in Fig. 2(b), and the displacement of the layer interface points coincident with the $z$ axis are shown in Fig. 2(c). Snapshots of the system at times $t$ $=0,0.45$, and $0.79 \mu$ s are shown in Fig. 3, corresponding to the initial state [part (a)], first [part (b)], and second [part (c)] collapses of the bubble. The curves for $z_{1}$ and $z_{2}$ shown in Fig. 2(c) correspond to the displacement of the points indicated by the black and white dots, respectively, in Fig. 3 from their initial state [Fig. 3(a)].

As Fig. 2 shows, maximum bubble translation occurs when the bubble radius is small. These points in time also correspond to peaks in the amplitudes of the surface modes $s_{2}$ and $s_{3}$, indicating the formation of a jet [Fig. 3(b)] pointing toward the center of the channel. An examination of Fig. 2(b) shows that the bubble tends to translate toward the

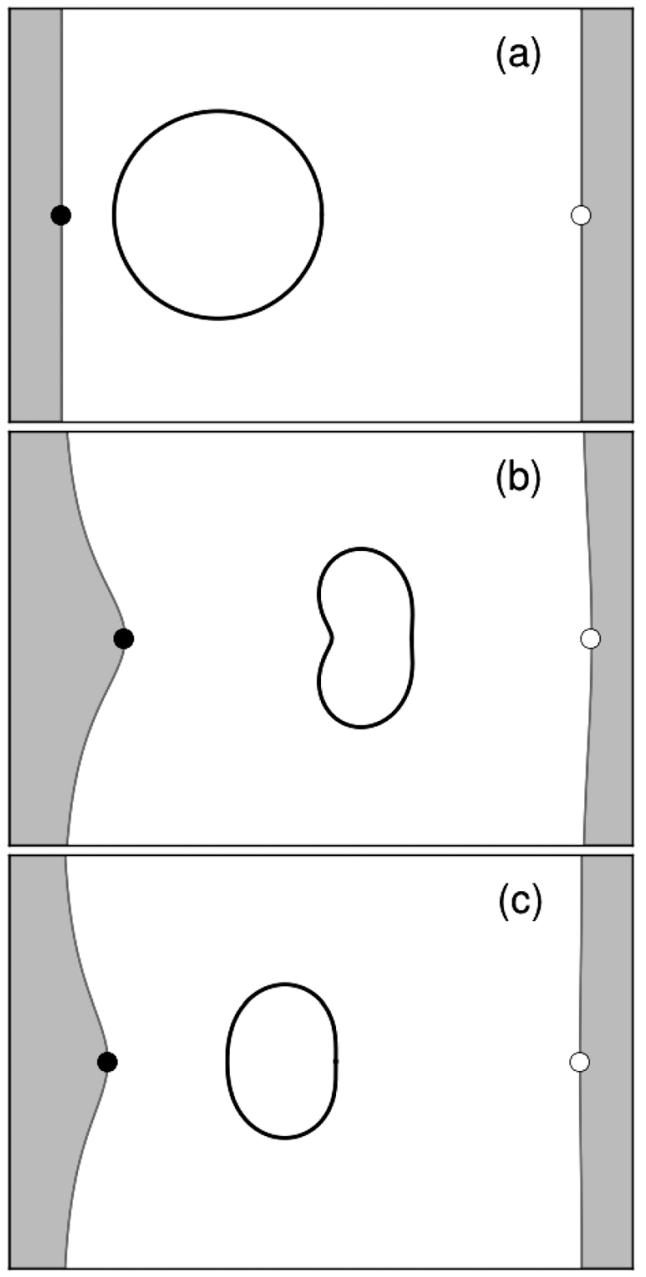

FIG. 3. System state at time equal to (a) 0 , (b) 0.45 , and (c) $0.79 \mu$ s for the simulation data plotted in Fig. 2.

center of the channel [dashed line at $Z / R_{0}=2.5$ in Fig. 2(b)]. This tendency may be understood by considering the initial translational force for a bubble initially spherical in shape and at rest. In this case $s_{n}, \dot{s}_{n}, \ddot{s}_{n}$, and $U$ are zero and $Z=Z_{0}$. Assuming small periodic spherical bubble pulsations, i.e., substituting

$$
R_{e}=R_{0}+\xi_{0} \sin \omega t, \quad \xi_{0} \ll R_{0},
$$

into Eq. (33) and averaging over one acoustic cycle yields

$$
\langle F\rangle=8 \pi \rho R_{0}^{4} \xi_{0}^{2}\left(\frac{\beta_{1}}{\left|Z_{0}-z_{1}\right|^{5}}-\frac{\beta_{2}}{\left|Z_{0}-z_{2}\right|^{5}}\right)+O\left(\xi_{0}^{4}\right)
$$

for the initial time-averaged translational force. Therefore, for $\beta_{1} \simeq \beta_{2}$ the translational force will tend to push the bubble toward the center of the channel, accompanied by jet formation in the same direction. It is interesting to note that the time-averaged translational force acting between a pulsating bubble and a rigid spherical particle of radius $R_{p}$ and density $\rho_{p}$ is $^{22,41}$

$$
\left\langle F_{\text {particle }}\right\rangle=4 \pi \rho \frac{R_{0}^{4} \xi_{0}^{2}}{d^{5}}\left(\frac{\rho-\rho_{p}}{\rho+2 \rho_{p}}\right) R_{p}^{3} \omega^{2},
$$




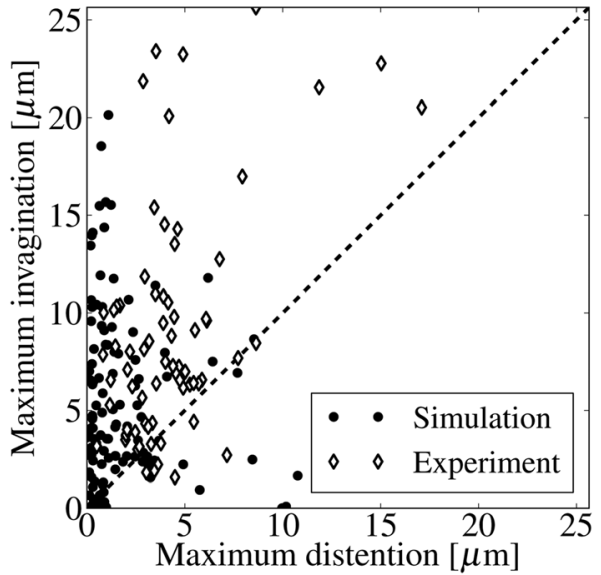

FIG. 4. Comparison of channel invagination and distention for 82 simulations (dots) chosen to correspond to the conditions in Fig. 2 of Ref. 29 (diamonds) due to the forced dynamics of a single spherical gas bubble with $R_{0}=1.5 \mu \mathrm{m}$. Each point represents the maximum inward or outward displacement of the layers for an individual simulation.

where $d$ is the distance between the centers of the bubble and particle, and has the same functional dependence on $R_{0}$, $\xi_{0}$ and $d$ (or $\left.\left|Z_{0}-z_{j}\right|\right)$ as indicated in Eq. (40). This is noteworthy since in the former case the force is due to a large, soft layer while in the latter case the bubble is influenced by a compact, rigid particle.

Note also in Figs. 2(c), 3(b), and 3(c) that the vessel walls have moved inward toward the center of the channel. These trends are consistent with laboratory measurements reported by Chen et al. ${ }^{29}$ who observed that maximum invagination of the vessel exceeded the accompanying distention in 60 of the 70 cases analyzed (85\%). In order to make direct comparisons with the results reported in Ref. 29 the dynamics of a single spherical translating gas bubble between the elastic layers is simulated over the range of channel widths and pressure amplitudes reported in the experimental study. Surface modes $s_{n}$ are neglected in these simulations because

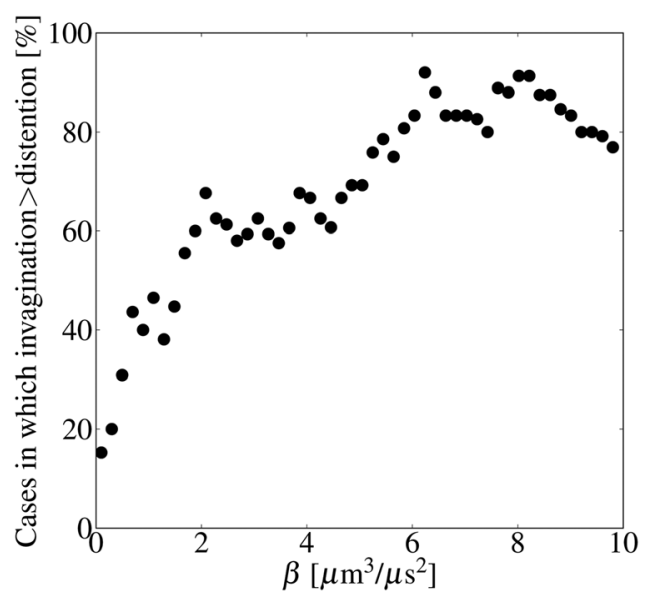

FIG. 5. Percent of cases in which invagination due to the dynamics of a bubble with equilibrium radius $1.5 \mu \mathrm{m}$ exceeded the associated distention for elastic layers having $\beta_{1}=\beta_{2}=\beta$ between 0.1 and $10 \mu \mathrm{m}^{3} / \mu \mathrm{s}^{2}$. One thousand simulations were run for each value of $\beta$ with channel widths ranging between 10 and $100 \mu \mathrm{m}$, pressure amplitudes between 0.8 and $7.2 \mathrm{MPa}$, and initial bubble-layer distances between 1.5 and $15 \mu \mathrm{m}$.

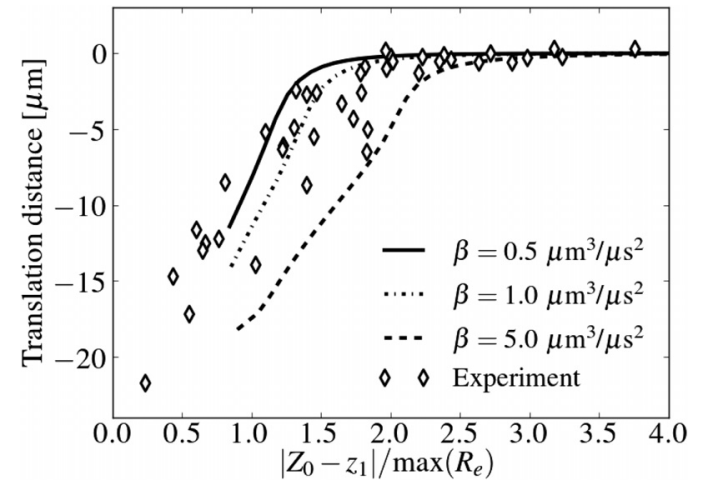

FIG. 6. Bubble translation distance as a function of normalized initial distance from the layer interface $\left|Z_{0}-z_{1}\right| / R_{e}$ for a bubble with equilibrium radius $R_{0}=2 \mu \mathrm{m}$ positioned in a channel of width $80 \mu \mathrm{m}$ and driven by two cycles of a sinusoidal pressure source with amplitude $1 \mathrm{MPa}$ and frequency $1 \mathrm{MHz}$. Results are shown for layers with $\beta=0.5,1$, and $5 \mu \mathrm{m}^{3} / \mu \mathrm{s}^{2}$. Simulation parameters were chosen to correspond to the experimental conditions reported in Fig. 4 of Ref. 30, replotted here (diamonds).

at these higher pressure amplitudes used in the experiments the surface mode amplitudes often become large, thereby violating the assumption that $\left|s_{n}\right| / R \ll 1$. The channel width is randomly chosen between 10 and $100 \mu \mathrm{m}$, and external pressure source amplitudes chosen between 0.8 and $7.2 \mathrm{MPa}$. In each case the excitation is two cycles of a sinusoidal pressure source with frequency $1 \mathrm{MHz}$. The elastic properties of the vessels and the initial offset distance between the bubble and the vessel wall were not reported in Ref. 29, so educated guesses are used to bound both of these parameters. In the simulations the value of $\beta$ is chosen between 5 and $10 \mu \mathrm{m}^{3} / \mu \mathrm{s}^{2}$, and the initial bubble-layer offset distances chosen between 1.5 and $15 \mu \mathrm{m}$. Each randomized parameter is chosen from a uniform distribution.

One thousand unique simulations were run and cases in which a bubble-layer collision occurred were discarded, with 82 simulations remaining for analysis. The values of $\beta, Z_{0}$, $W$ and drive pressure amplitude in these remaining cases span the complete ranges for each parameter. The maximum values of invagination and distention are shown by the black dots in Fig. 4. Data from the experiments reported in Ref. 29 are plotted with diamonds. Of the 82 simulations,

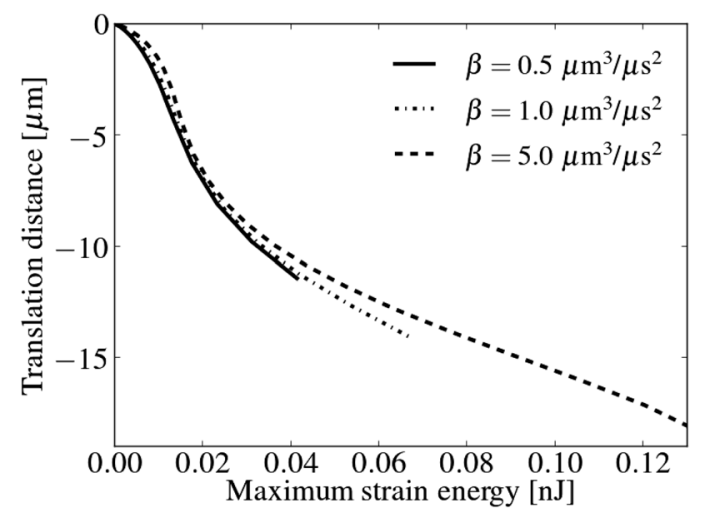

FIG. 7. Bubble displacement distance as a function of shear strain energy for the cases considered in Fig. 6. 
invagination is larger than distension in 60 , or $73 \%$, of the cases, which is comparable to the experimental observations.

However, the likelihood of invagination being greater than distention is dependent on the value of $\beta$. To illustrate this point, the simulations described above, and reported in Fig. 4, are repeated for various fixed values of $\beta$. One thousand simulations were performed for each value of $\beta$, with $Z_{0}, W$, and the acoustic pressure amplitude chosen randomly over the same ranges. The number of cases in which maximum invagination exceeded the maximum distention was recorded for each value of $\beta$ and plotted in Fig. 5. The simulations suggest that invagination may be expected to exceed distention if $\beta$ is greater than approximately $2 \mu \mathrm{m}^{3} / \mu \mathrm{s}^{2}$.

Finally, simulations are compared with the measurements of microbubble translation reported by Chen et al. ${ }^{30}$ in their Fig. 4. The dynamics of a spherical translating bubble with equilibrium radius $2 \mu \mathrm{m}$ between two elastic layers forming a channel of width $80 \mu \mathrm{m}$ was simulated in response to two cycles of a sinusoidal pressure source of amplitude $1 \mathrm{MPa}$, and frequency $1 \mathrm{MHz}$. Surface modes $s_{n}$ were neglected in this case for the same reasons cited in the discussion of Fig. 4. For elastic layers with $\beta=0.5$, 1 , or $5 \mu \mathrm{m}^{3} / \mu \mathrm{s}^{2}$ the initial distance of the bubble from the nearest layer $\left|Z_{0}-z_{1}\right|$ was varied from $3 R_{0}$ to $15 R_{0}$ and the maximum translation distance is determined in each case. Figure 6 shows the translation distance as a function of the initial standoff distance normalized by the maximum radius attained by the bubble, $\left|Z_{0}-z_{1}\right| / \max \left(R_{e}\right)$, and experimental observations from Ref. 30 are also plotted. Note that the general trend observed in experiments is captured in the simulations. The experimental points for which $\left|Z_{0}-z_{1}\right| / \max \left(R_{e}\right)$ $<1$ are difficult to simulate because a collision between the bubble and layer often occurs in these cases. However, the slopes of the three simulated curves is consistent with the experimental measurements taken for $\left|Z_{0}-z_{1}\right| / \max \left(R_{e}\right)<1$. The data in Fig. 6 show that for a given initial standoff distance the bubble will translate more for a larger value of $\beta$.

Some insight into this trend can be gained by examining the strain energy. As Eq. (17) shows, the strain energy is proportional to $\beta$ and therefore the bubble should translate farther for larger $\beta$. Figure 7 shows the translation distance for the same cases considered in Fig. 6 plotted instead as a function of the maximum value of the strain energy stored in the layers. Note that the three curves for different values of $\beta$ nearly coincide, suggesting that translation is chiefly a function of the shear strain energy stored in the vessel walls. The right endpoints of the three curves plotted in Fig. 7 correspond to the left endpoints in Fig. 6 near $\left|Z_{0}-z_{1}\right| / \max \left(R_{e}\right)=1$.

\section{CONCLUSIONS}

Dynamical equations for the motion of a pulsating and translating gas bubble with small shape deformations in a channel formed between two parallel elastic layers are derived using the Lagrangian formalism. Quadrupole and octupole spherical harmonics are included to model deformation of the bubble surface. An expression for the liquid velocity at the interface between the liquid and the layers yields an estimate of the layer displacement in response to the bubble motion. Simulations performed with the model equations show that a bubble initially positioned off center in the channel will tend to translate toward the center while initiating a jet in the direction of translation. The direction of jet formation in the simulations is qualitatively consistent with experimental observations. Simulations of bubble translation and vessel motion are compared to experimental measurements and quantitative agreement is demonstrated.

\section{ACKNOWLEDGMENTS}

This work was supported by the Postdoctoral Fellow Program at Applied Research Laboratories (T.A.H.), and NIH Grant Nos. DK070618 and EB011603. The authors thank Wayne Kreider, Thomas J. Matula, and Hong Chen at the Applied Physics Laboratory of the University of Washington for sharing the experimental data plotted in Figs. 4 and 6, and for comments and suggestions which improved the manuscript tremendously.

${ }^{1}$ Lord Rayleigh, "On the pressure developed in a liquid on the collapse of a spherical bubble," Philos. Mag. 34, 94-98 (1917).

${ }^{2}$ T. B. Benjamin and A. T. Ellis, "The collapse of cavitation bubbles and the pressures thereby produced against solid boundaries," Philos. Trans. R. Soc. London, Ser. A 260, 221-240 (1966).

${ }^{3} \mathrm{~W}$. Lauterborn and H. Bolle, "Experimental investigations of cavitationbubble collapse in neighborhood of a solid boundary," J. Fluid Mech. 72, 391-399 (1975)

${ }^{4}$ G. L. Chahine, "Interaction between an oscillating bubble and a free surface," J. Fluids Eng. 99, 709-716 (1977).

5 J. R. Blake and D. C. Gibson, "Growth and collapse of a vapor cavity near free surface," J. Fluid Mech. 111, 124-140 (1981).

${ }^{6}$ J. R. Blake and D. C. Gibson, "Cavitation bubbles near boundaries," Annu. Rev. Fluid Mech. 19, 99-123 (1987).

${ }^{7}$ Q. Wang, K. Yeo, B. Khoo, and K. Lam, "Nonlinear interaction between gas bubble and free surface," Comput. Fluids 25, 607-628 (1996).

${ }^{8}$ E. A. Brujan, K. Nahen, P. Schmidt, and A. Vogel, "Dynamics of laserinduced cavitation bubbles near an elastic boundary," J. Fluid Mech. 433, 251-281 (2001).

${ }^{9}$ S. W. Fong, E. Klaseboer, C. K. Turangan, B. C. Khoo, and K. C. Hung, "Numerical analysis of a gas bubble near bio-materials in an ultrasound field," Ultrasound Med. Biol. 32, 925-942 (2006).

${ }^{10} \mathrm{~K}$. Tachibana, T. Uchida, K. Ogawa, N. Yamashita, and K. Tamura, "Induction of cell-membrane porosity by ultrasound," Lancet 353, 1409 (1999).

${ }^{11} \mathrm{P}$. Marmottant and S. Hilgenfeldt, "Controlled vesicle deformation and lysis by single oscillating bubbles," Nature 423, 153-156 (2003).

${ }^{12}$ P. Prentice, A. Cuschieri, K. Dholakia, M. Prausnitz, and P. Campbell, "Membrane disruption by optically controlled microbubble cavitation," Nat. Phys. 1, 107-110 (2005).

${ }^{13}$ G. Taraldsen, "A note on reflection of spherical waves," J. Acoust. Soc. Am. 117, 3389-3392 (2004).

${ }^{14}$ A. A. Doinikov, L. Aired, and A. Bouakaz, "Acoustic response from a bubble pulsating near a fluid layer of finite density and thickness," J. Acoust. Soc. Am. 129, 616-621 (2011).

${ }^{15}$ A. A. Doinikov, L. Aired, and A. Bouakaz, "Acoustic scattering from a contrast agent microbubble near and elastic wall of finite thickness," Phys. Med. Biol. 56, 6951-6967 (2011).

${ }^{16}$ A. A. Doinikov and A. Bouakaz, "Theoretical investigation of shear stress generated by a contrast microbubble on the cell membrane as a mechanism for sonoporation," J. Acoust. Soc. Am. 128, 11-19 (2010).

${ }^{17}$ T. A. Hay, Yu. A. Ilinskii, E. A. Zabolotskaya, and M. F. Hamilton, "Model for bubble pulsation in liquid between parallel viscoelastic layers," J. Acoust. Soc. Am. 132, 124-137 (2012).

${ }^{18} \mathrm{H}$. N. Oguz and A. Prosperetti, "The natural frequency of oscillation of gas bubbles in tubes," J. Acoust. Soc. Am. 103, 3301-3308 (1998).

${ }^{19} \mathrm{E}$. Sassaroli and K. Hynynen, "Forced linear oscillations of microbubbles in blood capillaries," J. Acoust. Soc. Am. 115, 3235-3243 (2004). 
${ }^{20}$ J. R. Blake, B. B. Taib, G. Doherty, and Y. Tomita, "Transient cavities near boundaries. Part 1. Rigid boundary," J. Fluid Mech. 170, 479-497 (1986).

${ }^{21}$ J. B. Freund, "Suppression of shocked bubble expansion due to tissue confinement with application to shock wave lithotripsy," J. Acoust. Soc. Am. 123, 2867-2874 (2008).

${ }^{22}$ T. A. Hay, M. F. Hamilton, Yu. A. Ilinskii, and E. A. Zabolotskaya, "Model of coupled pulsation and translation of a gas bubble and rigid particle," J. Acoust. Soc. Am. 125, 1331-1339 (2009).

${ }^{23}$ Y. T. Hu, S. P. Qin, T. Hu, K. W. Ferrara, and Q. Jiang, "Asymmetric oscillation of cavitation bubbles in a microvessel and its implications upon mechanisms of clinical vessel injury in shock-wave lithotripsy," Int. J. Non-Linear Mech. 40, 341-350 (2005).

${ }^{24}$ T. Ye and J. L. Bull, "Microbubble expansion in a flexible tube," J. Biomech. Eng. 128, 554-563 (2006).

${ }^{25}$ S. Qin and K. W. Ferrara, "Acoustic response of compliable microvessels containing ultrasound contrast agents," Phys. Med. Biol. 51, 5065-5088 (2006).

${ }^{26}$ S. Qin and K. W. Ferrara, "The natural frequency of nonlinear oscillation of ultrasound contrast agents in microvessels," Ultrasound Med. Biol. 33, 1140-1148 (2007)

${ }^{27}$ C.-T. Hsiao, X. Lu, and G. Chahine, "Three-dimensional modeling of the dynamics of therapeutic ultrasound contrast agents," Ultrasound Med. Biol. 36, 2065-2079 (2010).

${ }^{28}$ C. F. Caskey, S. M. Stieger, S. Qin, P. A. Dayton, and K. W. Ferrara, "Direct observations of ultrasound microbubble contrast agent interaction with the microvessel wall," J. Acoust. Soc. Am. 122, 1191-1200 (2007).

${ }^{29}$ H. Chen, W. Kreider, A. A. Brayman, M. R. Bailey, and T. J. Matula, "Blood vessel deformations on microsecond time scales by ultrasonic cavitation," Phys. Rev. Lett. 106, 034301 (2011).

${ }^{30}$ H. Chen, A. A. Brayman, W. Kreider, M. R. Bailey, and T. J. Matula, "Observations of translation and jetting of ultrasound-activated microbubbles in mesenteric microvessels," Ultrasound Med. Biol. 37, 2139-2148 (2011).
${ }^{31}$ Yu. A. Ilinskii, M. F. Hamilton, and E. A. Zabolotskaya, "Bubble interaction dynamics in Lagrangian and Hamiltonian mechanics," J. Acoust. Soc. Am. 121, 786-795 (2007).

${ }^{32}$ E. Kurihara, T. A. Hay, Yu. A. Ilinskii, E. A. Zabolotskaya, and M. F. Hamilton, "Model for the dynamics of two interacting axisymmetric spherical bubbles undergoing small shape oscillations," J. Acoust. Soc. Am. 130, 3357-3369 (2011).

${ }^{33}$ S. Y. Emelianov, M. F. Hamilton, Yu. A. Ilinskii, and E. A. Zabolotskaya, "Nonlinear dynamics of a gas bubble in an incompressible elastic medium," J. Acoust. Soc. Am. 115, 581-588 (2004).

${ }^{34}$ A. A. Doinikov, "Translational motion of a bubble undergoing shape oscillations," J. Fluid Mech. 501, 1-24 (2004).

${ }^{35}$ E. A. Zabolotskaya, Yu. A. Ilinskii, G. D. Meegan, and M. F. Hamilton, "Modifications of the equation for gas bubble dynamics in a soft elastic medium," J. Acoust. Soc. Am. 118, 2173-2181 (2005).

${ }^{36}$ P. Marmottant, S. van der Meer, M. Emmer, M. Versluis, N. de Jong, S. Hilgenfeldt, and D. Lohse, "A model for large amplitude oscillations of coated bubbles accounting for buckling and rupture," J. Acoust. Soc. Am. 118, 3499-3505 (2005).

${ }^{37}$ A. A. Doinikov, J. F. Haac, and P. A. Dayton, "Modeling of nonlinear viscous stress in encapsulating shells of lipid-coated contrast agent microbubbles," Ultrasonics 49, 269-275 (2009).

${ }^{38}$ A. J. Reddy and A. J. Szeri, "Coupled dynamics of translation and collapse of acoustically driven microbubbles," J. Acoust. Soc. Am. 112, 1346-1352 (2002).

${ }^{39}$ J. Magnaudet and D. Legendre, "The viscous drag force on a spherical bubble with a time-dependent radius," Phys. Fluids 10, 550-554 (1998).

${ }^{40}$ M. P. Brenner, S. Hilgenfeldt, and D. Lohse, "Single-bubble sonoluminescence," Rev. Mod. Phys. 74, 425-484 (2002).

${ }^{41}$ W. T. Coakley and W. L. Nyborg, "Cavitation dynamics of gas bubbles," in Applications in Ultrasound: Its Applications in Medicine and Biology, edited by F. J. Fry (Elsevier, New York, 1978), pp. 77-159. 